\title{
Myelin Sheath Regeneration
}

National Cancer Institute

\section{Source}

National Cancer Institute. Myelin Sheath Regeneration. NCI Thesaurus. Code C161567.

Formation of new myelin sheaths on demyelinated axons. 